\title{
Biochemical Sensing Assays based on Coalescence- induced Self-propulsion Digital Microfluidics
}

\author{
Volker Nock, Yannick Muller, Mathieu Sellier \\ University of Canterbury \\ Christchurch, New Zealand \\ volker.nock@,canterbury.ac.nz
}

\author{
Claude Verdier \\ Laboratoire Interdisciplinaire de Physique \\ CNRS and University Joseph Fourier \\ Grenoble, France
}

\begin{abstract}
This work reports on coalescence-induced selfpropulsion as a driving mechanism to actuate microfluidic droplet assays. We demonstrate multi-droplet translation and assay-type sensing on a digital microfluidics platform by use of surface tension gradients alone. These gradients arise during the coalescence of two droplets of liquid having different compositions and therefore surface tensions. We demonstrate a chemiluminescence blood-detection reaction based on the mixing of two carrier droplets containing solutions of synthetic blood and luminol sensor solution. Presence of iron in the blood solution is recorded using digital imaging and analyzed via offline image processing. The results demonstrate the capability of the propulsion mechanism to propel droplets over several millimeters, thus enabling one to design a new family of chip-based biochemical sensor assays.
\end{abstract}

Microfluidics; sensor assay; chemiluminescence; luminol; image processing.

\section{INTRODUCTION}

Digital microfluidics is an open surface liquid-handling technology based on the manipulation of discreet picoliter- to microliter-sized droplets [1]. As opposed to closed-channel systems digital microfluidic devices offer unique advantages, such as simple instrumentation, flexible device geometry, and easy coupling with other external technologies. In particular, the simple liquid handling and controlled sample volumes in these devices have led to increasing interest in the integration of biochemical assays onto such platforms [2]. Due to the open transparent nature of the platforms, optical analyte detection via chemiluminescence sensor systems can be readily achieved. A wide variety of biologically relevant compounds can be detected using such dissolved sensors when coupled with an optical signal acquisition setup [3]. Currently, most existing digital microfluidic platforms rely on the use of electrode arrays or surface acoustic waves for droplet translation on-chip. This significantly increases the device fabrication complexity and thus limits the platform applicability.

Based on our work to model the coalescence of sessile droplets [4], we have recently introduced the concept of using the coalescence of two miscible, partially wetting droplets with different surface tensions as a novel droplet propulsion method [5]. A theoretical model based on the lubrication approximation framework, combined with numerical simulation results predicted the occurrence of self-propulsion behavior. To confirm the phenomenon we performed a parametric study to explore the role of two dimensionless numbers on the propulsion speed and total displacement. As a direct outcome of this study it was found that the resulting surface tension gradient gives rise to Marangoni flow, as illustrated in Fig. 1, which, if sufficiently large, can displace the droplet on a substrate [5].

We experimentally demonstrated the propulsion mechanism for a variety of different fluid combinations and developed an improved model based on measured droplet velocities [6]. Largest lateral translations were observed for aqueous/ethanol (carrier/fuel) droplet combinations, however, the exact nature of the water/ethanol interplay and propulsion mechanism for this system are not yet fully understood [7]. Our above mentioned open-surface experiments were facilitated by guiding droplets within micro-patterned hydrophilic traces on a flat, hydrophobic polydimethylsiloxane (PDMS) substrate. This setup and choice of materials make the mechanism directly applicable to digital microfluidics.

In this paper we thus explore the use of such plasmapatterned traces and coalescence-induced droplet propulsion to demonstrate spatially-controlled multi-droplet actuation and mixing for the chemiluminescent detection of blood. The
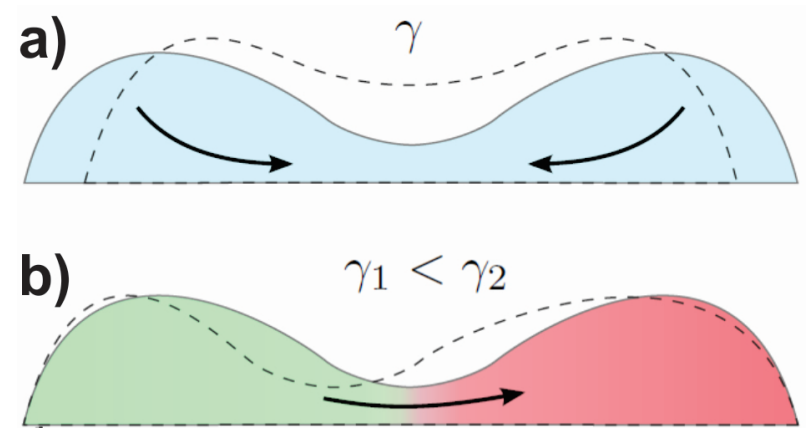

Figure 1. Mechanism of coalescence-induced self-propulsion: (a) Coalescence of two sessile droplets of the same liquid and surface tension $\gamma$. Laplace pressure, much lower in the neck region, "sucks" liquid from the surrounding volume (black arrows), thus growing the neck over time (dashed line). (b) Coalescence of droplets with different surface tensions $\gamma_{1}<\gamma_{2}$. Upon contact Marangoni stress induced flow occurs, draining liquid from one droplet to the other (left to right). This results in net motion of the combined droplet towards the larger surface tension region. 
experiments shown in the following illustrate the potential of this phenomenon to yield a new family of digital microfluidic assay platforms.

\section{EXPERIMENTAL}

Hydrophilic traces on PDMS substrates were prepared by micro-milling of CAD-designed channel outlines into flat polystyrene (PS) cell-culture well lids (NUNC) using a Micromill CNC Mini-Mill/GX with $300 \mu \mathrm{m}$ cutting tools. These PS stencils were then used to transfer the desired traces onto precast flat PDMS substrates by exposure to RF oxygen plasma. Flat PDMS substrates were initially prepared by mixing Sylgard 184 (Dow Corning) base:curing agent at a 10:1 w/w ratio. The pre-polymer mixture was then degassed using a desiccator and poured to a typical thickness of $5 \mathrm{~mm}$ into single-well PS cell culture tray bottoms. After $2 \mathrm{~h}$ at $80^{\circ} \mathrm{C}$ curing on a hotplate, PDMS sheets were peeled out of the wells and cured for an additional $2 \mathrm{~h}$ at $80^{\circ} \mathrm{C}$ for further crosslinking and to stabilize the surface properties. Prepared samples were left at room temperature for a minimum of $24 \mathrm{~h}$ before use.

Machined PS stencils containing the through-hole channel outlines were then placed into intimate contact with the PDMS surface. The stack was loaded into a barrel plasma etcher (Emitech K1050X, Quorum) and exposed to $\mathrm{O}_{2}$ plasma for $10 \mathrm{~s}$ at $100 \mathrm{~W}$ RF power setting. After removal from the plasma chamber the PS stencil was removed from the PDMS and the substrates were ready for use. Due to the temporary nature of the PDMS surface modification [8], substrates were experimented on immediately after fabrication. Up to eight parallel traces of various lengths and with mixing areas at various distances were incorporated into the design. All of the fabricated designs included on a single stencil could be actuated simultaneously on the PDMS substrate.

For experimentation, samples were placed on a flat bench in a standard laboratory environment and propulsion "fuel", sensor and analyte solution droplets were placed manually onto the substrate using an electronic multi-channel micropipette (0.2$10 \mu 1$ Picus, Biohit). Registration marks on the stencil allowed for marking and visual identification of the starting points of the invisible hydrophilic traces on the PDMS. Fig. 2 shows a photograph of the PS stencil and optical micrographs of the resulting PDMS substrates during testing with dye-colored water droplets and $70 \%$ ethanol as "fuel". Millimeter paper was placed underneath the PDMS substrates to provide a scale reference. Droplet translation was recorded was recorded continuously using a high-speed digital camera (MQ013MG-E2, Ximea) connected to a PC via USB-3 interface. Recorded sequences were analyzed using the Image Processing toolbox included in Matlab (R2012b, Mathworks).

To demonstrate bio-sensing on chip, aqueous sensor droplets containing latent bloodstain reagent [9] and an analyte sample droplet containing a synthetic blood analog (Bluestar Forensic) were propelled and subsequently mixed using ethanol fuel droplets. Chemiluminescence emitted from the reaction of the bloodstain with iron catalyst in the blood analog solution was recorded in a dark room using the same digital camera system as described above. The luminescence intensity over time was analyzed by converting the recorded video file to individual frames. This image sequence was then imported into Matlab.
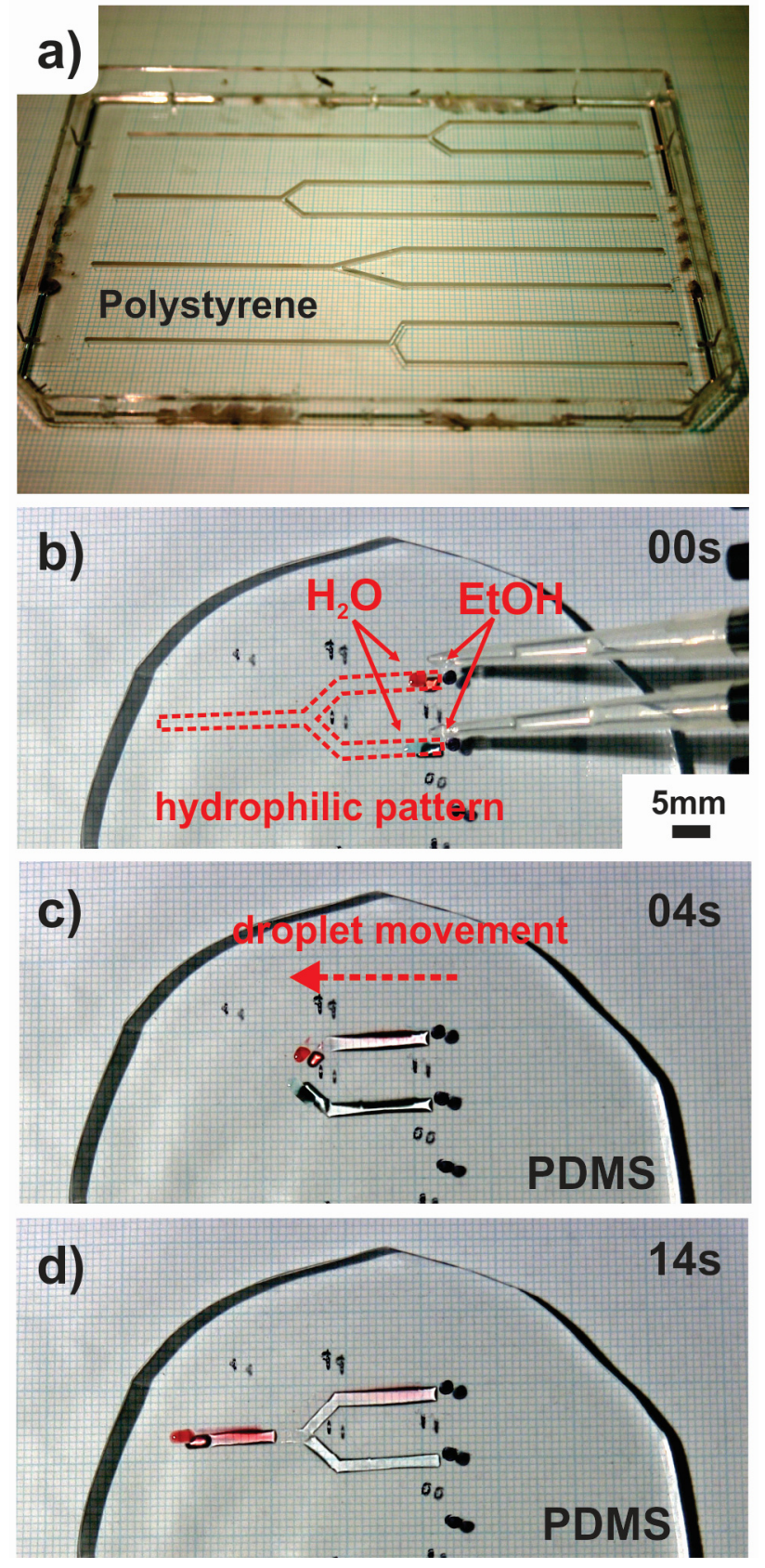

Figure 2. Coalescence-driven, surface chemistry-guided droplet propulsion on plasma activated PDMS substrates: (a) Photograph of a typical polystyrene stencil used to plasma-treat the thermally cured PDMS substrates. Traces visible in the PS correspond to milled through-holes used to render PDMS hydrophilic. (b) Optical micrograph showing the hydrophilic pattern (dashed outline) on the PDMS surface and placement of sample (red and blue dye colored $\mathrm{H}_{2} \mathrm{O}$ ) and fuel $(\mathrm{EtOH})$ droplets using a micropipette. (c) Optical micrograph of the same sample $4 \mathrm{~s}$ after coalescence showing red and blue droplets during lateral translation close to trace junction. The dashed arrow indicates the direction of droplet movement. (d) Same sample imaged $14 \mathrm{~s}$ after initiation of propulsion. Sample droplets have mixed and continue on-chip in a single channel to the end of the pre-patterned hydrophilic trace. 
Using the Image Processing Toolbox a region of interest was defined and the luminescence intensity averaged within this area and plotted versus time.

\section{RESULTS AND DISCUSSION}

The droplet propulsion and mixing capabilities of the platform were first demonstrated by translating multi-colored water droplets on a PDMS surface. A multi-channel pipette was used to place two differently colored, water-based 'carrier' droplets onto each pre-patterned channel trace. This was followed by the deposition of two ethanol 'fuel' droplets in close proximity to each of the aqueous carrier droplets. As both pairs of droplets spread within their respective hydrophilic trace, neck formation and coalescence are initiated. Fig. 2(b) shows optical micrographs of the placement of the fuel ethanol droplets next to the colored water droplets with invisible hydrophilic traces indicated by dashed lines superimposed onto the PDMS substrate. Upon coalescence of the carrier and fuel droplets the carrier droplets propel to the left, guided by the hydrophilic traces on the surface.

After $4 \mathrm{~s}$ of translation, as shown in Fig. 2(c), droplets have moved approximately $18 \mathrm{~mm}$ across the substrate, including a change of direction via a $45^{\circ}$ angle patterned into each trace. This angle culminates in a trace junction where the two traces converge into a single trace of equal width, thus mixing the carrier droplets. Depending on the geometrical design of the trace junction the resulting mixed aqueous droplet can be stopped or guided further, as shown in Fig. 2(d). For the junction design introduced in Fig. 2 the mixed droplet continues a further $18 \mathrm{~mm}$ to a final translation distance of $36 \mathrm{~mm}$ at $14 \mathrm{~s}$ since application of the fuel droplets. This demonstrates the platforms ability to successfully perform digital microfluidic-type liquid handling operations and staggered multi-solute mixing.

To further illustrate the applicability of the platform to biochemical assay-type sensing, we performed a chemiluminescence blood-detection reaction on-chip. The principle of the experimental setup, based on the mixing of two carrier droplets containing solutions of synthetic blood and a luminol-like sensor, is shown schematically in Fig. 3. Using prepatterned hydrophilic traces similar in design to those shown in Fig. 2, each droplet is translated approximately $25 \mathrm{~mm}$ to a circular mixing area by coalescence with an ethanol fuel droplet. At the mixing point the presence of iron compounds in the synthetic blood droplet is detected by reaction with the sensor solution. This reaction emits blue luminescence (wavelength $430 \mathrm{~nm}$ ), which is recorded via an external imaging setup.

An example of such a detection experiment is shown in Fig. 4. The complete droplet translation and biochemical sensing sequence is also available online as an annotated video (http://youtu.be/WIQZYlHTkCk). Initially under normal laboratory light conditions, as displayed in Fig. 4(a), the synthetic blood and bloodstain reagent droplets on two independent trace-lines can be observed translating on the PDMS surface. Both droplets guided by the top two traces are about to enter the downstream mixing area. A second two-trace layout located underneath the first only shows the sensor droplet entering its mixing area. In this case the synthetic blood droplet was deliberately blocked using a hydrophobic barrier to a)

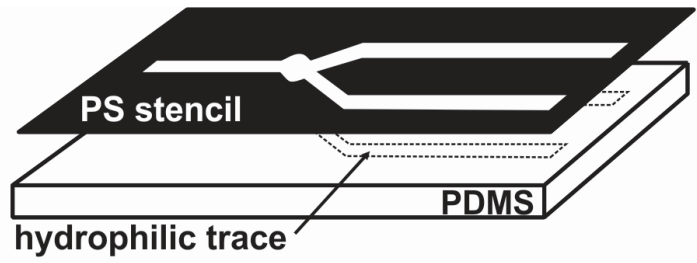

b)

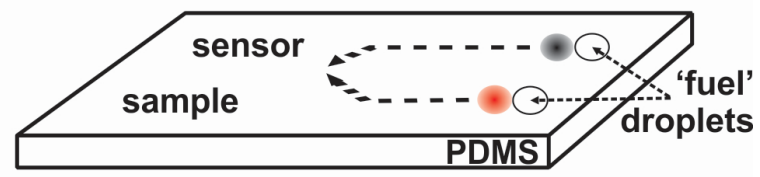

c)

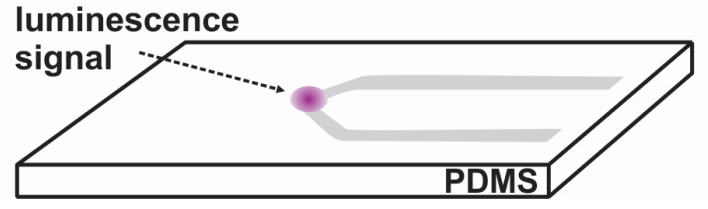

Figure 3. Schematic of the on-chip blood detection assay: (a) Plasma generation of hydrophilic traces on a flat PDMS substrate using micro-milled PS stencils. (b) Placement of the sample/sensor and fuel droplets by micro-pipetting to initiate propulsion. (c) Detection of sample content via luminescence reaction upon droplet translation and mixing at a downstream trace junction.

visualize the difference in resulting intensity when analyte and sensor droplets do not meet in the mixing area.

Once ambient light is turned off, the luminescence resulting from the mixing of the two droplets in the top part of Fig. 4(b) becomes apparent. Similar to the reaction with standard luminol, this luminescence indicates the presence of an oxidizing agent and a transition metal. In this case the main components capable of catalyzing this reaction for emitting light are the transition metals haem and peroxidase [10]. Haem is a biochemical structure that forms an integral part of peroxidase. This structure is equally present in hemoglobin. In this manner, the presence of hemoglobin, or blood, can be revealed by taking advantage of the ability of haem to catalyze the chemo-luminescent property of the luminol compound.

Fig. 4(c) shows a plot of the resulting luminescence intensity over time as recorded in the two mixing areas. In the presence of an analyte droplet containing synthetic blood the intensity increases significantly compared to the second, non-mixing set of droplets. This clearly demonstrates the suitability of the platform for on-chip assay-type reactions and biochemical sensing. While the initial sensor response increases rapidly, several step changes can be observed in the sensor response until the intensity finally levels out at close to $6 \mathrm{~s}$ after coalescence. These variations in intensity increase are attributed to the mixing dynamics of the system. While studying the propulsion mechanism, we have observed that droplets propelled on plasma-patterned PDMS regularly exhibit a velocity profile with variations on a similar time scale [6] to the ones seen here. Initial observations suggests that this might be due to local surface variations in the polymer which could be overcome by extended thermal aging prior to use [8]. 


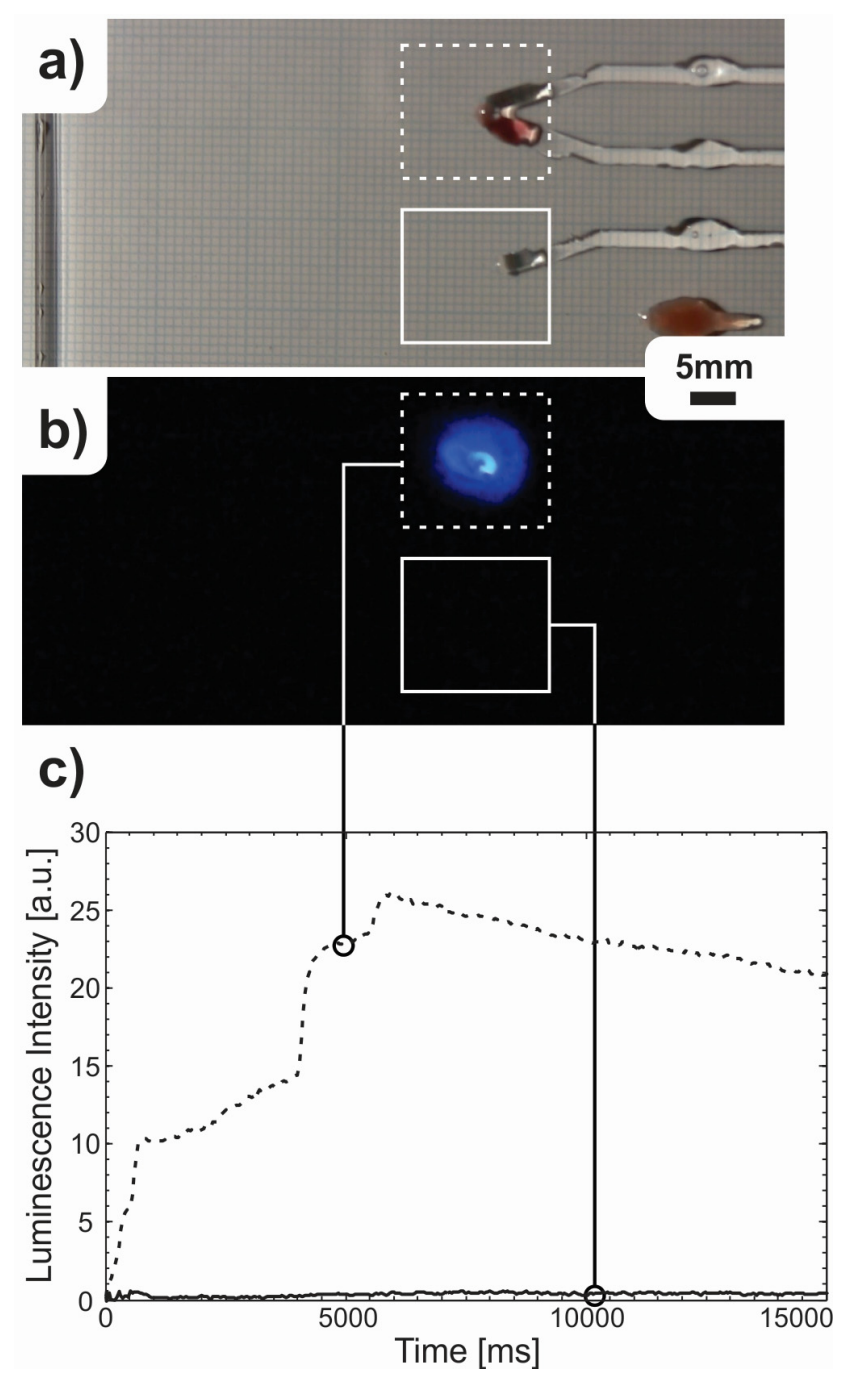

Figure 4. Blood detection assay: (a) Optical micrograph showing two sets of EtOH-propelled droplets containing luminol and synthetic blood translating on a PDMS substrate. The luminol droplet in the bottom trace was blocked using a hydrophobic barrier (non-plasma treated PDMS). (b) Optical micrograph of the same area after ambient light was switched off. The luminescence signal distribution represents a single frame during mixing of droplets at the junction. No luminescent response is observed in the lower channel due to blocked luminol droplet. (c) Plot of average luminescence intensity within the indicated regions from onset of mixing at the junction $(0 \mathrm{~ms})$ to the full recording time of $15 \mathrm{~s}$. Step-like variations in the intensity are indicative of mixing and reaction dynamics.

After detection the mixed droplet resulting from the top two traces in Fig. 4 was observed to remain trapped inside the circular mixing area for the remainder of the recording. In contrast to this, the single analyte droplet in the third trace, which did not mix with its respective sensor droplet blocked in the bottom trace, continued unimpeded through its similarlyshaped mixing area. This indicates that the geometry of the mixing area, as well as mixing, can be used to influence the continuation of droplet translation. In the future we are aiming to replace plasma-patterning with chemical surface patterning to produce the guidance traces. This should reduce the observed variations in the propulsion effect and will also extend the overall applicability of the platform by increasing the long-term stability of the hydrophilic treatment [11]. In addition, we are currently evaluating a wide variety of other luminescence-based sensing systems relevant to biochemical applications. In particular, this effort is focused on cancer detection using the presence of ATP. In combination with existing, fully-automated robotic dispensing technology this will enable a whole new family of chip-based assays.

\section{CONCLUSION}

We have demonstrated a new digital microfluidics sensing platform based on the coalescence-induced self-propulsion of droplets. Droplet translation, mixing and a biochemical sensing reaction were demonstrated on-chip. The suitability of the platform for biochemical assays was demonstrated by reaction of a luminol-based sensor solution with synthetic blood inside carrier droplets. Resulting sensor luminescence was recorded and analyzed using an external digital imaging setup. The results indicate the suitability of the platform and propulsion mechanism for droplet microfluidics-based reaction control and general biochemical assays.

\section{ACKNOWLEDGMENTS}

The authors gratefully acknowledge the support of the Royal Society of New Zealand through a Marsden Grant (Grant number UOC1104) and the MacDiarmid Institute for Advanced Materials and Nanotechnology for sponsoring a studentship. Technical support was provided by Helen Devereux and Gary Turner.

\section{REFERENCES}

[1] K. Choi, A. H. Ng, R. Fobel and A. R. Wheeler, "Digital microfluidics", Annu. Rev. Anal. Chem., vol. 5, pp. 413-440, 2012.

[2] L. Malic, D. Brassard, T. Veres and M. Tabrizian, "Integration and detection of biochemical assays in digital microfluidic LOC devices", Lab Chip, vol. 10, pp. 418-431, 2010.

[3] H. A. J. Al Lawati, "Flow-based analysis using microfluidicschemiluminescence systems", Luminescence, in print.

[4] M. Sellier and E. Trelluyer, "Modeling the coalescence of sessile droplets", Biomicrofluid., vol. 3, pp. 022412-022414, 2009.

[5] M. Sellier, V. Nock, and C. Verdier, "Self-propelling, coalescing droplets", Int. J. Multiphase Flow, vol. 37, pp. 462-468, 2011.

[6] M. Sellier, V. Nock, C. Gaubert, and C. Verdier, "Droplet actuation induced by coalescence: Experimental evidences and phenomenological modeling”, Eur. Phys. J. ST, vol. 219, pp. 131-141, 2013.

[7] P. Bahadur, P. S. Yadav, K. Chaurasia, A. Leh and R. Tadmor, "Chasing drops: Following escaper and pursuer drop couple system", J. Coll. Interf. Sci., vol. 332, pp. 455-460, 2009.

[8] J. Zhou, A. V. Ellis and N. H. Voelcker, "Recent developments in PDMS surface modification for microfluidic devices“, Electrophoresis, vol. 31, pp. 2-16, 2010.

[9] L.J Blum, P. Esperança, S. Rocquefelte, "A new high-performance reagent and procedure for latent bloodstain detection based on Luminol chemiluminescence", Can. Soc. Forensic Sci. J., vol. 39, pp. 81-100, 2006.

[10] S. S. Tobe, N. Watson and N. N. Daéid, : Evaluation of Six Presumptive Tests for Blood, Their Specificity, Sensitivity, and Effect on High Molecular-Weight DNA", J. Forensic Sci., vol. 52, pp. 102-109, 2007.

[11] S. Hemmilä, J. V. Cauich-Rodríguez, J. Kreutzer and P. Kallio, "Rapid, simple, and cost-effective treatments to achieve long-term hydrophilic PDMS surfaces”, Appl. Surf. Sci., vol. 258, pp. 9864-9875, 2012. 